\title{
Effects of spatially directed attention on visual encoding
}

\author{
MARK TIPPENS REINITZ \\ Southeastern Louisiana University, Hammond, Louisiana
}

\begin{abstract}
The purpose in this study was to distinguish among possible mechanisms by which focused attention facilitates visual perceptual processing in a cued discrimination task. In two experiments, subjects verified the presence of an X in masked, briefly presented, four-letter arrays. On most trials, subjects were precued to the location of the stimulus array (valid-cue condition); however, sometimes a nonstimulus location was cued (invalid-cue condition). The exposure duration of the stimulus array was varied. In Experiment 1, there was a large effect of cue condition on hit probability, but no effect of cue condition on false alarm probability. In Experiment 2, there was a large effect of cue condition on $d^{\prime}$. In both experiments, the stimulus duration needed to reach any given performance level was greater by a constant factor for stimuli in the invalid-cue than it was in the valid-cue condition. This suggests that visual information is acquired (or utilized) more rapidly from attended than from unattended locations.
\end{abstract}

Many variables affect the processes responsible for the acquisition of visual perceptual information from briefly presented stimuli. These include not only perceptual variables such as stimulus luminance (Loftus, 1985) and masking (Loftus, Johnson, \& Shimamura, 1985) but also highlevel cognitive variables. As an instance of the latter, Reinitz, Wright, and Loftus (1989) have demonstrated that semantic priming directly affects the acquisition of perceptual information from pictures. This article addresses the role of another cognitive variable in the acquisition of perceptual information from briefly presented visual stimuli; specifically, focused visual attention.

In many experiments, it has been demonstrated that performance is better for attended visual stimuli than it is for unattended visual stimuli across a wide variety of tasks. In such experiments, a brief cue prior to the presentation of the stimulus array serves as a signal for subjects to expect a target stimulus in a specific spatial location. Researchers using this procedure have demonstrated better performance for validly than for invalidly cued stimuli across a number of tasks, including simple detection (e.g., Bashinski \& Bacharach, 1980; Posner, Snyder, \& Davidson, 1980), identification (e.g., C. W. Eriksen \& Hoffman, 1972; van der Heijden, Wolters, Groep, \& Hagenaar, 1987), and discrimination (e.g., Downing, 1988), as well as across the two dependent measures of

\footnotetext{
Many thanks to Geoff Loftus for his numerous comments and suggestions. Bob Dale, Charles Eriksen, Jeff Miller, Tram Neill, and an anonymous reviewer also provided many helpful comments on an earlier version of the manuscript. I am grateful to Dorothy Taylor for running the subjects and for her many helpful comments. The research was supported by a faculty development grant from Southeastern Louisiana University to Mark T. Reinitz; Experiment 1 was presented at the 1988 Psychonomic Society meeting in Chicago. Correspondence may be addressed to Mark T. Reinitz, Department of Psychology, Box 831, Southeastern Louisiana University, Hammond, LA 70402.
}

response latency (e.g., Jonides, 1980; Posner, 1980) and accuracy (e.g., Downing, 1988; Prinzmetal, Presti, \& Posner, 1986). The effect of the cue is independent of eye movements, and it occurs when the cued and uncued locations are equidistant from fixation (Jonides, 1980; Posner, 1980; Posner et al., 1980).

At what processing level does attention exert its effect? Some of the earlier studies cited above were ambiguous in this regard; their results could be attributed equally well to perceptual processes or to postperceptual decision processes (for a discussion, see Prinzmetal et al., p. 361). However, more recent studies leave little doubt that the locus of the effect is perceptual (cf. Downing, 1988; Prinzmetal et al., 1986). For instance, a recent study by Downing (1988) provides compelling evidence for a role of spatially directed attention in visual perceptual processing. She found that sensitivity (measured as $d^{\prime}$ ) was greatest for stimuli presented to cued locations, and that it decreased with distance from the cued location. In separate experiments, cuing facilitated form, orientation, brightness, and luminance discriminations. Downing (1988) concluded that focused attention affects the quality of perceptual representations by affecting the pick-up of information from attended areas.

\section{Purpose of the Current Research}

The purpose in the present study was to investigate the specific mechanism by which focused attention facilitates visual perceptual processing. In the experiments reported here, the stimuli were rectangular arrays of four letters, which contained either four Os or three Os and an X. On most trials, the subjects were precued to the location of the array; on some trials, an invalid location was cued. The stimulus arrays were presented for various exposure durations and followed by a mask; accuracy at detecting an $\mathrm{X}$ was measured. 
There are two general ways in which attention could facilitate encoding in such an experiment. By an additive account, the cue provides information that otherwise would have to be acquired from the stimulus itself; the cue is therefore equivalent to a brief presentation of the stimulus array. By a multiplicative account, the cue increases the rate at which information from the target is encoded. These two alternatives are discussed in turn.

\section{Additive Models of Attentional Effects}

By an additive account, the encoding process is initiated by the cue and thus begins before stimulus presentation. A valid-cue condition therefore provides a head start in processing relative to an invalid-cue condition, by providing information that otherwise could not be acquired until the stimulus array was present. For instance, a number of authors have proposed that encoding requires the alignment of an attentional "spotlight" with the stimulus array (see Posner et al., 1980; Remington \& Pierce, 1984; Shulman, Remington, \& McLean, 1979; Tsal, 1983). For valid cues, this alignment can occur prior to stimulus presentation. When the cue is invalid, however, subjects must locate the stimulus and shift attention while the stimulus is present. The valid-cue condition thus provides $k$ additional milliseconds to encode stimulus information relative to the invalid-cue condition, where $k$ represents the time to locate and move attention to the array in the invalid-cue condition.

I refer to the function relating the detectability of an $\mathrm{X}$ in the stimulus array (measured, e.g., as $d^{\prime}$ ) to stimulus exposure duration, $t$, as a performance curve. By an additive account, the function relating performance curves for a valid- and an invalid-cue condition is

$$
P V(t)=P I(t+k),
$$

where $P V(x)$ and $P I(x)$ denote performance in a valid- and an invalid-cue condition following exposure duration $x$, and $k$ represents the equivalent exposure duration contributed by the valid cue. For all performance levels, any performance level attained for a $t$-millisecond stimulus presentation in a valid-cue condition will be attained for a $(t+k)$-millisecond stimulus presentation in an invalidcue condition. Therefore, there is a constant $k$-millisecond separation between the two performance curves; I describe the curves as horizontally parallel. This prediction is graphically presented in Figure 1, top panel.

\section{Multiplicative Models of Attentional Effects}

By a multiplicative account, the effect of attending to a stimulus location is to increase the rate at which visual information is acquired from that location relative to unattended locations. For example, Downing (1988) proposes that attention exerts its effect by facilitating the pickup of perceptual information from the attended location; hence more information is acquired per unit time from attended locations than it is from unattended locations. By a multiplicative account, the equation relating perfor- mance curves for a valid- and an invalid-cue condition is

$$
P V(t)=P I(c t),
$$

where $c$ is the factor by which attention increases rate of encoding. Here the performance curves diverge horizontally, as is shown in Figure 1 (middle panel). The nature of the divergence is quite specific: Stimuli preceded by an invalid cue require an exposure that is longer by some factor, $c$, relative to validly cued stimuli, to achieve any performance level. The predictions of this alternative can be conveniently tested by plotting response accuracy as a function of log, rather than linear, time. Because equal ratios in linear coordinates correspond to equal distances in log coordinates, the prediction of the multiplicative model is that the performance curves for the valid- and invalid-cue conditions are horizontally parallel when plotted as a function of log exposure duration. The equation relating the two performance curves is then

$$
P V[\ln (t)]=P I[\ln (c)+\ln (t)] \text {. }
$$

The hypothetical data shown in the middle panel of Figure 1 are replotted on a log exposure-duration axis in the bottom panel. The performance curves are now horizontally parallel.
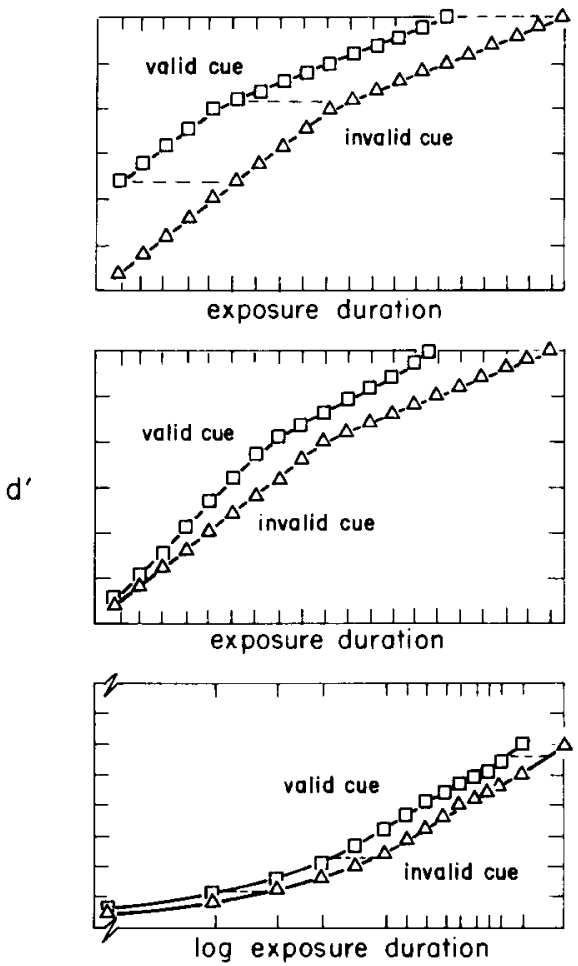

Figure 1. Hypothetical data showing the predictions of the two models. The top panel shows the additive model prediction: Performance curves are horizontally parallel on a linear exposure-duration axis. The middle and bottom panels show the multiplicative prediction: Performance curves diverge on a linear exposure-duration axis (middle panel) and are horizontally parallel on a log exposureduration axis (bottom panel). 
Previous Applications of the Analysis Technique

The predictions I have been describing constitute a specific application of a general analysis technique for discriminating between additive and multiplicative models. This technique, which was first described in the classic work of Stiles (1939), has more recently been used by Loftus and his colleagues to study visual encoding of pictures. For instance, Loftus et al. (1985) used it to investigate visual encoding from iconic memory. Masked and unmasked pictures were presented for varying exposure durations. The additive model was confirmed: Performance curves for the masking conditions were horizontally parallel on a linear duration axis. The additive constant separating the curves was about $100 \mathrm{msec}$; the conclusion was that an icon is equivalent to an additional $100 \mathrm{msec}$ of stimulus presentation. Loftus (1985) also performed a series of experiments in which the luminance and exposure duration of target pictures were varied. In this case, the multiplicative model was confirmed: When memory performance for high- and low-luminance pictures was plotted as a function of log exposure duration, the two curves were separated by a horizontal constant, indicating that increasing luminance serves to increase the rate at which information is acquired from pictures. Most recently, Reinitz et al. (1989) applied the technique to study the effects of semantic priming on visual encoding of pictures; the finding was that, for short stimulus exposures, primes exert a multiplicative effect on encoding, and for long stimulus exposures, primes exert an additive effect.

\section{Generality of the Analysis Technique}

In the experimental paradigm with which I will be concerned, validly and invalidly cued stimulus arrays are shown at varying exposure durations and followed by a mask. Additive cue-effect models predict performance curves for the valid- and invalid-cue conditions to be horizontally parallel on a linear duration axis. Multiplicative models predict performance curves for these conditions to be horizontally parallel on a log duration axis. These predictions are invariant over all one-to-one transformations of the dependent variable (see Loftus, 1978); since they involve comparisons of stimulus durations that produce equal performance, any pair of points that are equal in one performance scale (e.g., $d^{\prime}$ ) must also be equal in a transformed scale (e.g., $\log d^{\prime}$ ). It is not necessary to assume, for instance, that equal increments in the amount of encoded information result in equal increments in performance for all performance levels. Neither is it necessary to make any strong assumptions about the nature of perceptual information. For instance, there is no need to assume that the information is acquired as a set of specific features. The only necessary assumption is that the dependent variable is monotonically related to the amount of information acquired from the stimulus. This assumption seems reasonable in the present case; since the present paradigm provides both hit and false alarm rates for the various experimental conditions, it is possible to use $d^{\prime}$ as the performance measure.
Finally, it is important to point out that the predictions of the additive and multiplicative models hold over a variety of potential subject strategies. For instance, it is not necessary to assume that subjects always attend to the cued location. Several studies have indicated that subjects typically attend to the cued location less than $100 \%$ of the time; instead, the frequency with which they attend to the cued location is roughly proportional to the probability that the cue is valid (C. W. Eriksen \& Yeh, 1985; Jonides, 1980 ). Such a strategy will affect only the value of $k$ (in an additive model) or $c$ (in a multiplicative model). The basic predictions of the models (i.e., the predictions of horizontally parallel performance curves on a linear or log exposure-duration axis) remain the same, regardless of whether or not the subjects use a probability-matching strategy (a proof of this is given in the Appendix).

\section{EXPERIMENT 1}

The purpose in Experiment 1 was to test between the additive and multiplicative accounts of the facilatory effects of focused visual attention.

\section{Method}

Subjects. One hundred and twenty Southeastern Louisiana University undergraduates participated for class credit. They were run individually.

Stimulus and Apparatus. The stimulus arrays contained either four Os or three Os and an X. They were arranged rectangularly, so that each array subtended about $95^{\circ}$ of visual angle vertically and $.85^{\circ}$ horizontally. The gap from fixation to the closest corner of the array subtended about $1.3^{\circ}$. The cue consisted of a greater than and a less than sign bracketing an array location. These pointed at one another, and were centered with respect to the array. The gap between them subtended about $1^{\circ}$ of visual angle. The mask was a set of dollar signs, which filled both rows of all array positions for each trial. Dollar signs were chosen because piloting indicated that they provided the most effective mask of any of the available computer-generated characters. Subjects viewed the stimuli from a distance of $320 \mathrm{~cm}$

The stimuli were presented on IBM 13-in. color monitors controlled by IBM PC computers. The software that ran the experiment ensured that each presentation (including the mask) was put on the screen in a single pass of the raster. The most accurate control of exposure duration possible on such an apparatus was achieved by using an internal timer with millisecond accuracy, and by continuously monitoring the raster position. This made it possible to begin writing to the monitor at the start of each pass.

Design and Procedure. Each subject received 240 experimental trials. Over these, the cue was valid two thirds of the time. On invalid trials, one of the uncued quadrants was randomly chosen as the stimulus location. The target $\mathrm{X}$ was present on half the trials; its location in the stimulus array was chosen randomly. The array exposure duration was $33,66,99,132$, or $165 \mathrm{msec}$. Cue validity, target presence or absence, and exposure duration were factorially combined, resulting in 20 experimental conditions. The 10 conditions for which the cue was valid occurred 16 times each over the 240 trials; each of the remaining conditions occurred 8 times. Condition order was random, and a different random order was used for each subject. Finally, arrays in each of the 20 conditions were presented equally often to each of the four quadrants.

The sequence of events for each trial was as follows: First, a fix ation dot appeared at the center of the screen and remained for $2 \mathrm{sec}$ The cue then appeared at one of the four quadrants for $99 \mathrm{msec}$ 
Table 1

Experiment 1: Hit and False Alarm Probabilities for the Five Exposure Durations in the Two Cuing Conditions

\begin{tabular}{lccccc}
\hline & \multicolumn{5}{c}{ Exposure Duration (milliseconds) } \\
\cline { 2 - 6 } Cuing Condition & 33 & 66 & 99 & 132 & 165 \\
\hline \multicolumn{5}{c}{ Hit Probabilities } \\
Valid cue & .672 & .711 & .769 & .785 & .783 \\
Invalid cue & .525 & .600 & .670 & .704 & .716 \\
& False Alarm Probabilities & & \\
Valid cue & .370 & .305 & .262 & .241 & .218 \\
Invalid cue & .334 & .273 & .253 & .240 & .234 \\
\hline
\end{tabular}

This was immediately followed by the stimulus array, which was presented for $33,66,99,132$, or $165 \mathrm{msec}$. The mask was then presented for $250 \mathrm{msec}$. The subjects indicated the presence or absence of the target by pressing keys on the computer keyboard. The fixation dot for the next trial appeared immediately after the response.

The instructions to the subjects stressed that upon presentation of the cue they were to direct attention to the cued location without moving their eyes from the fixation point. These instructions to maintain fixation were repeated three times, and all the subjects indicated that they understood them. The subjects had 80 practice trials. After that, the subjects were asked whether they had moved their eyes during the trials. Most said they had not; a few said that they had made eye movements on the first few trials, but that the task seemed easier when they kept their eyes stationary. The subjects were reminded of the importance of maintaining fixation before receiving the experimental trials.

\section{Results and Discussion}

Table 1 presents the mean probabilities of correctly responding that an $\mathrm{X}$ was present (hit rate) and incorrectly responding that an $\mathrm{X}$ was present (false alarm rate) for the various conditions. Separate ANOVAs were performed on the hit and false alarm data. For the hit data, there were extremely reliable effects of both cue validity $[F(1,119)=64.536, p<.001]$ and exposure duration $[F(4,476)=49.236, p<.001]$. The interaction was also significant $[F(4,476)=3.328, p<.05]$. For false alarms, only the main effect of exposure duration was significant; false alarms decreased with increasing exposure duration $[F(4,476)=33.392]$. False alarm rates did not differ between cue conditions $[F(1,119)=1.648, p>.1]$. Orienting attention to the stimulus array increases correct detections but does not produce an increase in false alarms. This clearly indicates that attention affects encoding.

In order to test whether the effect of attention on encoding is additive or multiplicative, it was first necessary to generate performance curves for the valid- and invalidcue conditions. These are presented in Figure 2 (left panel): $d^{\prime}$ is plotted as a function of exposure duration for the two cue conditions. The crucial test of the two explanations involved determining whether a constant horizontal difference existed between these two conditions when they were plotted on a linear or a logarithmic axis of stimulus duration. The curves were replotted on both a linear exposure-duration axis and a log exposureduration axis. The curve for the valid-cue condition was then shifted horizontally on both axes by the mean horizontal distance between the curves; horizontally parallel curves will overlap following such a shift. The results of these horizontal shifts are presented in the middle and right panels of Figure 2. A horizontal shift on a log axis shows more overlap than does a horizontal shift on a linear exposure duration axis. This leads to the tentative conclusion that visual information is encoded more rapidly from attended than from unattended locations.

\section{EXPERIMENT 2}

The conclusion that attention speeds encoding depends on the finding of better overlap between performance curves when they are shifted on a log rather than on a linear exposure-duration axis. There is, unfortunately, no convenient statistical test to demonstrate that the log shift
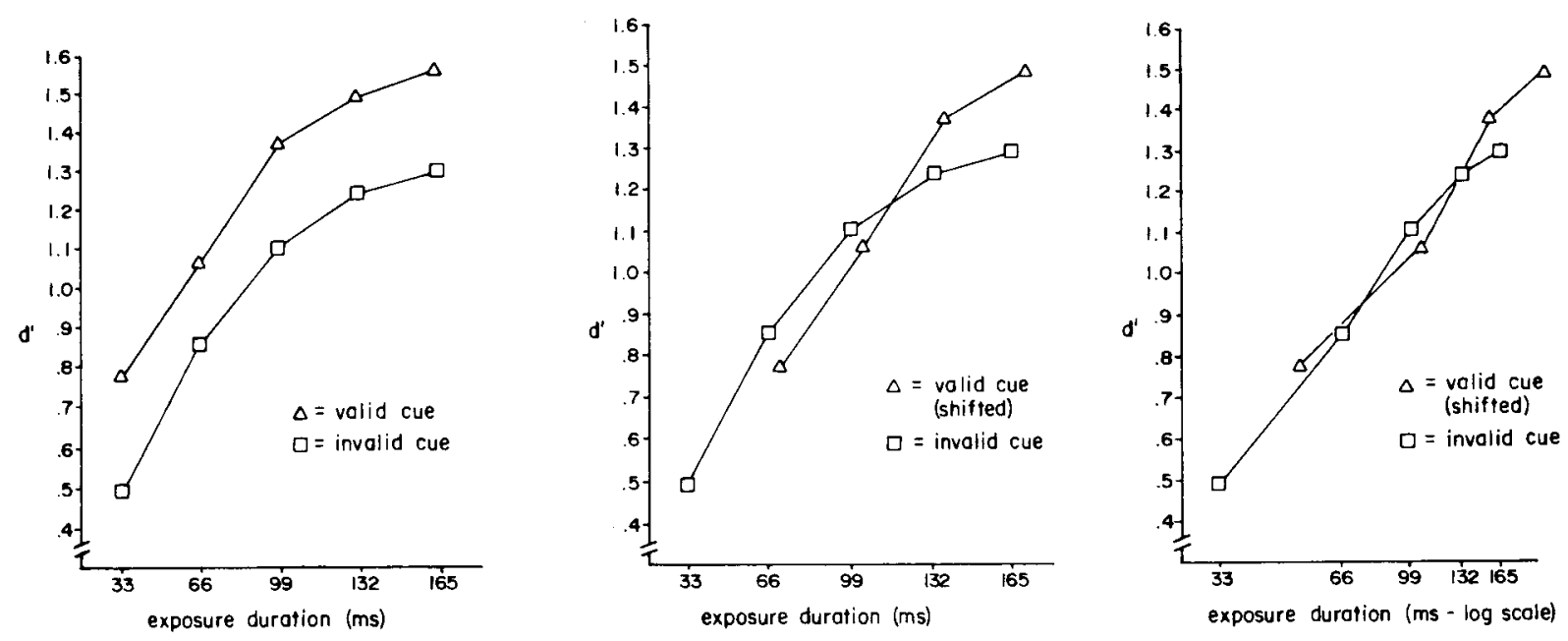

Figure 2. Experiment 1: Performance curves for the valid- and invalid-cue conditions. In the left panel, $d^{\prime}$ is plotted as a function of exposure duration. In the middle and right panels, time is on a linear and log scale, respectively, and the valid-cue curve has been shifted horizontally to provide the best overlap. 
produces significantly better overlap than the linear shift does. An alternative approach is to show that the log shift produces better overlap across successive replications of the experiment. Experiment 2 provides such a replication.

There are several important differences between Experiments 1 and 2. First, in Experiment 1, there were relatively few trials per subject. As a result, most subjects had false alarm rates of 0 for at least a few conditions. It was therefore necessary to calculate $d$ 's from group means, making it impossible to calculate the standard error for the performance curves presented in Figure 2. Furthermore, it was necessary to perform ANOVAs on hit and false alarm data, rather than on $d^{\prime}$. For this reason, the conclusion that attention facilitates encoding is statistically weak. In Experiment 2, these problems were corrected by greatly increasing the number of trials per subject. Finally, the total number of observations per condition was increased in order to produce stable performance-curve estimates.

\section{Method}

Subjects. Forty-eight Southeastern Louisiana University undergraduates participated for class credit. They were run individually.

Stimulus and Apparatus. The stimuli and apparatus were the same as in Experiment 1.

Design and Procedure. Each subject participated in four experimental sessions spaced 1 week apart. In each session, there were 80 practice trials, followed by 240 experimental trials. The target $X$ was present on half the trials. The exposure durations were the same as in Experiment 1. Location cues were valid two thirds of the time. Cue validity, target presence or absence, and exposure duration were again factorially combined, resulting in 20 experimental conditions. The 10 conditions for which the cue was valid occurred 16 times each in a given session, and the 10 invalid-cue conditions occurred 8 times each. A different random condition order was used for each session. Finally, arrays in each of the conditions were presented equally often to each quadrant.
The sequence of events for each trial was the same as in Experiment 1 , and the precautions relevant to eye movements taken in Experiment 1 were repeated for Experiment 2.

\section{Results and Discussion}

In this experiment, it was possible to calculate $d^{\prime}$ for each condition for each subject. These were averaged to generate the performance curves for the two cue conditions shown in Figure 3 (left panel). An ANOVA performed on the $d^{\prime}$ values showed reliable effects of cue condition $[F(1,47)=34.832, p<.01]$ and exposure duration $[F(4,188)=104.362, p<.01]$. The interaction was also significant $[F(4,188)=5.822, p<.01]$.

The relationship between the valid- and invalid-cue performance curves is of primary interest. In Figure 3, these have been shifted on both linear (middle panel) and log (right panel) exposure-duration axes in order to provide the best overlap. As was the case for the Experiment 1 data, the linear shift provides poor overlap, disconfirming the additive prediction. The overlap produced by the $\log$ shift is fairly dramatic. As in Experiment 1, the multiplicative prediction is confirmed. Taken together, the results of Experiments 1 and 2 indicate that focused attention speeds the acquisition of visual information from the stimulus.

\section{GENERAL DISCUSSION}

\section{Implications of the Results}

Additive models predict that performance curves for the valid- and invalid-cue conditions should be horizontally parallel on a linear exposure-duration axis. This prediction was disconfirmed. Several authors have proposed additive explanations of the role of attention in detection experiments (Posner et al., 1980; Remington \&
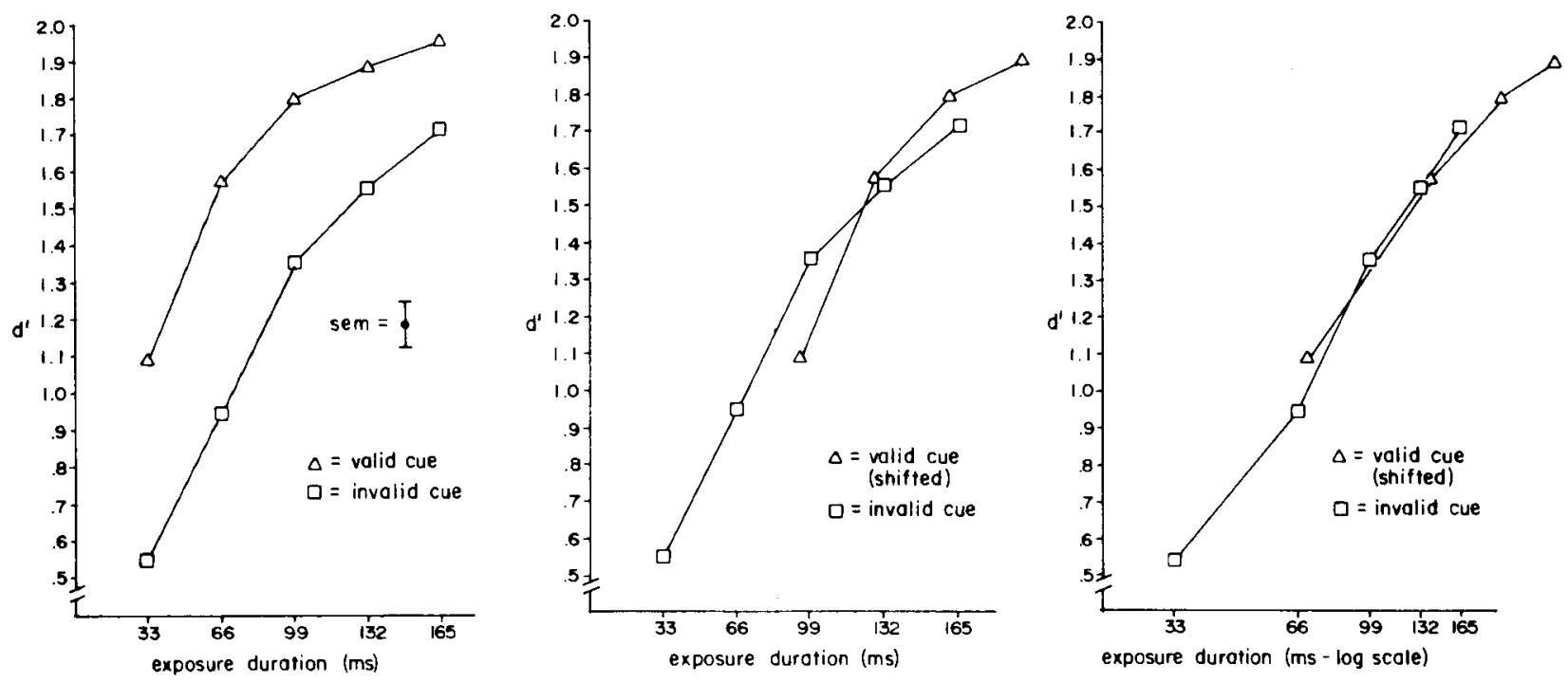

Figure 3. Experiment 2: Performance curves for the valid- and invalid-cue conditions. In the left panel, $d$ ' is plotted as a function of exposure duration. In the middle and right panels, time is on a linear and log scale, respectively, and the valid-cue curve has been horizontally shifted to provide the best overlap. 
Pierce, 1984; Shulman et al., 1979; Tsal, 1983). These authors argue that longer detection latencies for targets in an invalid- rather than a valid-cue condition are due to the time needed to move an attentional "beam' from the cued to the target location when the cue is invalid. In the present experiments, the performance deficit observed in the invalid-cue condition cannot have been due to additional time required to align an attentional mechanism with the target. The data clearly indicate that attention affects encoding rate, rather than simply the onset of processing.

Simple spotlight theories of visual attention have come under attack in recent years. Several authors have pointed out methodological flaws in experiments that have purported to provide evidence for an attentional spotlight (see, e.g., C. W. Eriksen \& Murphy, 1987). Furthermore, other recent experimental findings are incompatible with an attentional spotlight (cf. Cheal \& Lyon, 1988; Driver \& Baylis, 1989; Murphy \& C. W. Eriksen, 1987). It is well known that the time to respond to a target letter decreases as the distance between the target and a distractor letter increases (B. A. Eriksen \& C. W. Eriksen, 1974). This has been taken as evidence for an attentional spotlight; the interfering effect of the distractor decreases because it leaves the bounds of spatial attention. However, Driver and Baylis (1989) have recently demonstrated that distant distractors that move with the target produce more interference than do stationary letters that are near a moving target. This finding is damaging to spotlight theories, and it indicates that we allocate attention to perceptual groupings. Cheal and Lyon (1988) found that it took their subjects no longer to shift attention $10^{\circ}$ than to shift attention only $2^{\circ}$ when the to-be-attended stimuli were matched for visibility. This finding is also damaging to spotlight theories. Thus the current study adds to a growing body of evidence against simple spotlight theories of attention.

Simple spotlight theories represent only one of a number of possible additive explanations ruled out by the present results. For instance, one might propose that spatial location is in itself a visual feature that must be encoded about a stimulus. According to this explanation, the effect of a valid cue is to provide a visual feature (spatial location) prior to stimulus presentation that otherwise would have to be encoded while the stimulus was present. This is clearly an additive explanation, and it is inconsistent with the present findings.

Multiplicative models of attention effects predict that performance curves for a valid- and an invalid-cue condition will diverge when plotted as a function of linear exposure duration, and that they will be horizontally parallel as a function of $\log$ exposure duration. This prediction was confirmed, indicating that visual information is acquired more rapidly from attended than from unattended locations. Several authors have offered models consistent with this finding. Downing (1988) has proposed that pick-up of perceptual information is most efficient at attended areas, and that it becomes increasingly less efficient as distance from the center of attention increases.
This implies a difference in the rate at which information is acquired from attended and unattended areas. Similarly, Murphy and C. W. Eriksen (1987) propose that a precue leads to facilitation for stimuli presented to the attended area, and, further, that there is a gradient of decreasing facilitation along the borders of focused attention. If facilitation takes the form of increased encoding rate, this model is also consistent with the current findings.

It should be pointed out that a purely multiplicative model does not necessitate relocation of attention to the target following an invalid cue in order for discrimination to occur. Thus, in employing the curve-shift methodology I have assumed that focal attention is not necessary for discrimination. There is substantial evidence to support this assumption: A large number of studies indicate that discrimination can occur with little or no attention devoted to the stimulus when the target does not share visual features with the distractors (see Folk \& Egeth, 1989; Treisman \& Gelade, 1980). It is likely that subjects in the present experiments did not focus the entirety of their attentional resources on the cued location. Rather, some attention was probably distributed across the remaining stimulus locations. Regardless of whether subjects paid no attention to the noncued locations, or simply paid less attention to those locations, the conclusion of increased processing rate for attended stimuli still follows.

\section{Alternative Accounts of the Results}

It is important to point out that the simple additive and multiplicative models described here do not exhaust all possible models of attentional facilitation. Hybrid models are possible that would include both additive and multiplicative effects. There is no evidence for this type of model in the present data (for a detailed discussion of hybrid models and their predictions, see Reinitz et al., 1989, pp. 292-294).

Other types of models exist that are neither additive nor multiplicative. For example, C. W. Eriksen and St. James (1986) have proposed a model in which attentional resources are reallocated over time. If the rate of encoding depends on the amount of attentional resource allocated to the stimulus, then encoding rate would be expected to increase over time as increasingly more attention is allocated to the stimulus. According to such a model, subjects begin to reallocate attention to the cued location at cue onset. If the cue is valid, stimulus encoding starts out at a rapid rate, since the subject has had the cue exposure to reallocate attentional resources. If it may be assumed that the subject will continue to allocate remaining resources to the stimulus location during stimulus exposure, encoding rate will continue to increase while the stimulus is present. On invalid-cue trials, subjects do not begin to allocate resources to the stimulus location until stimulus onset. At stimulus onset, encoding rate is thus relatively slow, but it increases during stimulus exposure as increasing resources are allocated to the stimulus location.

The specific predictions of this resource-allocation model will be determined by the function describing the 
change in encoding rate with increasing resourceallocation time, which is unknown. Figure 4 shows the predictions of the model for the simplest possible assumptions; specifically, that encoding rate is 0 when time available to allocate resources is 0 , and that the change in encoding rate with increasing resource-allocation time is 1 (arbitrary) unit of information per millisecond per millisecond of resource-allocation time. These assumptions make it possible to generate a curve relating encoding rate (in arbitrary units of information per millisecond) to resource-allocation time. If $t^{\prime}$ is time available to allocate resources, the amount of information acquired (in arbitrary units) for various validly and invalidly cued stimulus exposures can be derived by calculating the area under the curve from $t^{\prime}$ at stimulus onset to $t^{\prime}$ at stimulus offset. Note that $t^{\prime}$ at stimulus onset is 0 for invalidly cued stimuli, but that it is equal to the exposure duration of the cue for validly cued stimuli. At stimulus offset, $t^{\prime}$ is equal to stimulus exposure duration for invalidly cued stimuli, and it is equal to stimulus exposure duration plus cue duration for validly cued stimuli.

The top panel of Figure 4 shows performance curves predicted for a valid- and an invalid-cue condition when cue duration is $100 \mathrm{msec}$. Information is scaled in arbitrary units; however, because I am interested in predicting horizontal relations between curves, and because horizontal relations are invariant over monotonic transformations, I am free to scale the ordinate in any way I choose. Although the curves diverge, they are not related by a multiplicative constant. The curves have been shifted on both a linear exposure-duration axis (middle panel) and a log exposure-duration axis (bottom panel). In this case, neither shift produces better overlap-which is to be expected, since the model is neither additive nor multiplicative. Clearly, different encoding rate change functions will result in different performance curves. Some of these might approximate the pattern of results obtained in Experiments 1 and 2 . However, in the absence of other evidence to distinguish this model from a simple multiplicative model, the latter should be preferred, for two reasons. First, the prediction of the multiplicative model is highly specific. In contrast, there exists no version of a resourceallocation model that makes an equally specific prediction. Second, the multiplicative model is quite simple, and it therefore provides the most parsimonious account of the data.

Another alternative explanation for the results needs to be considered: If subjects moved their eyes to the stimuli, the results might be due to more rapid processing in the fovea than in the periphery of the retina. Since eye movements were not monitored, this explanation for the results cannot be ruled out completely. However, for all but the longest exposure durations, the total time elapsed from the onset of the cue to the offset of the stimulus array was too short for a subject to initiate and carry out an eye movement. A robust effect of cue validity was found for even the shortest exposure durations. In fact, in Experiment 2 , the largest absolute performance difference between the valid- and invalid-cue conditions occurred for
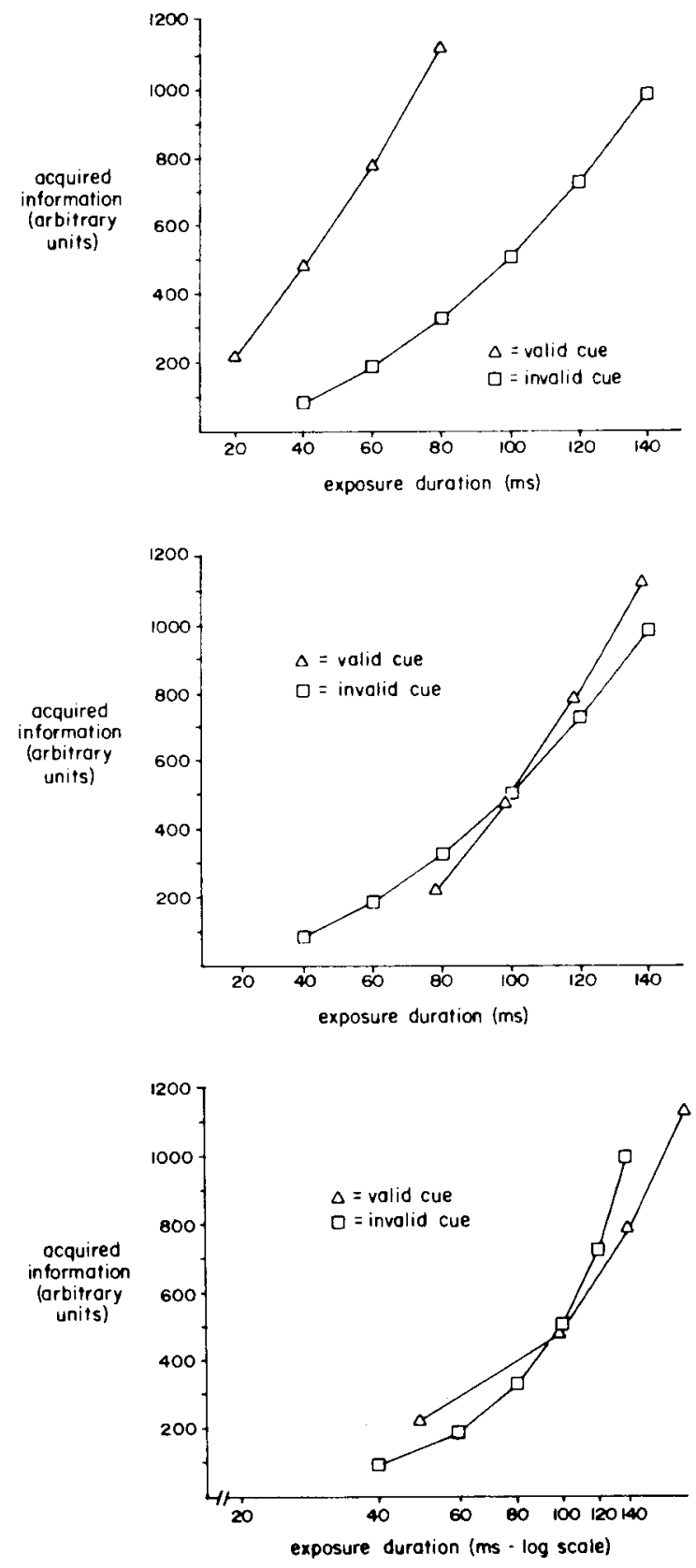

Figure 4. Performance curves predicted by one version of a resource-allocation model. The curves are shifted on a linear (middle panel) and log (bottom panel) exposure-duration axis to provide the best overlap.

the 33-msec exposures. This duration was certainly too brief to permit eye movements. In any case, a variety of experiments have demonstrated that the facilitation provided by the cue is not due to eye movements (see Posner, 1980; Posner et al., 1980). Thus it is unlikely that the results are due to eye movements.

\section{Closing Comments}

In closing, it is of interest that the experiments reported here demonstrate that spatial information presented prior 
to a stimulus speeds encoding. Reinitz et al. (1989) have demonstrated that providing semantic information prior to a stimulus also speeds stimulus encoding, at least for relatively short stimulus durations. Priming effects are ostensibly quite different from the cue effects reported here. However, both are cases in which prior presentation of stimulus-relevant information increases the rate of stimulus encoding. It is plausible that a single underlying mechanism is responsible for both types of effects. Other types of cues have been shown to facilitate detection performance as well; detection is facilitated by prior knowledge of form (Teichner \& Krebs, 1974), spatial frequency (Davis, Kramer, \& Graham, 1983), and color (Humphreys, 1981). All of these variables may exert their effects by focusing attention on task-relevant stimulus information, thereby increasing the rate at which these features are encoded.

\section{REFERENCES}

BASHINSKI, H. S., \& BACHARACH, V. R. (1980). Enhancement of perceptual sensitivity as the result of selectively attending to spatial locations. Perception \& Psychophysics, 28, 241-248.

Cheal, M. L., \& Lyon, D. R. (1988, November). Evidence against a moving spotlight theory of visual attention. Paper presented at the Twenty-Ninth Annual Meeting of the Psychonomic Society, Chicago.

Davis, E. T., Kramer, P., \& Graham, N. (1983). Uncertainty about spatial frequency, spatial position, or contrast of visual patterns. Perception \& Psychophysics, 33, 20-28.

DownING, C. J. (1988). Expectancy and visual-spatial attention: Effects on perceptual quality. Joumal of Experimental Psychology: Human Perception \& Performance, 14, 188-202.

Driver, J., \& BAyLIS, G. C. (1989). Movement and visual attention: The spotlight metaphor breaks down. Journal of Experimental Psychology: Human Perception \& Performance, 15, 448-456.

Eriksen, B. A., ERIKSEN, C. W. (1974). Effects of noise letters upon the identification of a target letter in a nonsearch task. Perception \& Psychophysics, 16, 143-149.

ERIKSEN, C. W., \& HoffMaN, J. E. (1972). Temporal and spatial characteristics of selective encoding from visual displays. Perception \& Psychophysics, 12, 201-204.

Eriksen, C. W., \& MURPHY, T. D. (1987). Movement of attentional focus across the visual field: A critical look at the evidence. Perception \& Psychophysics, 42, 299-305.

ERIKSEN, C. W., \& ST. JAMES, J. D. (1986). Visual attention within and around the field of focal attention: A zoom lens model. Perception \& Psychophysics, 40, 225-240.

ERIKSEN, C. W., \& YEH, Y. (1985). Allocation of attention in the visual field. Joumal of Experimental Psychology: Human Perception \& Performance, 11, 583-597.

FolK, C. L., \& EGETH, H. (1989). Does the identification of simple features require serial processing? Journal of Experimental Psychology: Human Perception \& Performance, 15, 97-110.

HumphreYs, G. W. (1981). Flexibility of attention between stimulus dimensions. Perception \& Psychophysics, 30, 291-302.

JoNIDES, J. (1980). Towards a model of the mind's eye's movement. Canadian Journal of Psychology, 34, 103-112.

LoFTus, G. R. (1978). On interpretation of interactions. Memory \& Cognition, 6, 312-319.

LoFTUs, G. R. (1985). Picture perception: Effects of luminance on available information and information-extraction rate. Joumal of Experimental Psychology: General, 114, 342-356.

Loftus, G. R., Johnson, C. A., \& Shimamura, A. P. (1985). How much is an icon worth? Journal of Experimental Psychology: Human Perception \& Performance, 11, 1-13.

MurPhy, T. D., \& ERIKSEN, C. W. (1987). Temporal changes in the distribution of attention in the visual field in response to precues. Perception \& Psychophysics, 42, 576-586.
PosNer, M. I. (1980). Orienting of attention. Quarterly Journal of Experimental Psychology, 32, 3-25.

Posner, M. I., Snyder, C. R. R, \& Davidson, B. J. (1980). Altention and the detection of signals. Journal of Experimental Psychology: General, 109, 160-174.

Prinzmetal, W., Presti, D. E., \& Posner, M. I. (1986). Does attention affect visual feature integration? Journal of Experimental Psychology: Human Perception \& Performance, 12, 361-369.

ReINITZ, M. T., Wright, E., \& LoFTUS, G. R. (1989). Effects of semantic priming on visual encoding of pictures. Journal of Experimental Psychology: General, 118, 280-297.

Remington, R. W., \& Pierce, L. (1984). Moving attention: Evidence for time-invariant shifts of visual selective attention. Perception \& Psychophysics, 35, 393-399.

Shulman, G. L., Remington, R. W., \& Mclean, J. P. (1979). Moving attention through visual space. Journal of Experimental Psychology: Human Perception \& Performance, 5, 522-526.

STILES, W. S. (1939). The directional sensitivity of the retina and the spectral sensitivities of the rods and cones. Proceedings of the Royal Society of London (Series B), 127, 64-105.

TeichNer, W. H., KREBS, M. J. (1974). Laws of visual choice reaction time. Psychological Review, 81, 75-98.

Treisman, A., \& GelaDE, G. (1980). A feature-integration theory of attention. Cognitive Psychology, 12, 97-136.

TSAL, Y. (1983). Movements of attention across the visual field. Journal of Experimental Psychology: Human Perception \& Performance, 9, 523-530.

van der Heijden, A. H. C., Wolters, G., Groep, J. C., \& HAGENAAR, R. (1987). Single-letter recognition accuracy benefits from advance cuing of location. Perception \& Psychophysics, 42, 503-509.

\section{APPENDIX}

\section{Proof That Additive Predictions Hold for a Probability-Matching Strategy}

Assume that attending to the stimulus location provides some equivalent exposure duration, $k$. Further assume that on some proportion of the trials, $j(j>.5)$, subjects attend to the cued location. If $t$ is the valid-cue exposure duration required to reach some performance level for trials on which subjects attend to the cue, then the average valid-cue exposure duration required to reach any given performance level is

$$
j t+(1-j)(t+k) \text {. }
$$

I assume that when a subject does not attend to the cued location, the subject is equally likely to attend to any of the remaining three locations. The proportion of invalid-cue trials for which subjects will attend to the location where the stimulus appears is then $(1-j) / 3$. Thus, for invalid-cue trials, the average exposure duration required for equivalent performance is

$$
\left(\frac{1-j}{3}\right) t+\left[1-\left(\frac{1-j}{3}\right)\right](t+k) \text {. }
$$

Let $m=(1-j) / 3.1-j$ then equals $3 m$. For any performance level, the difference between valid- and invalid-cue exposure durations predicted by the additive model is then

$$
\begin{aligned}
{[m t+(1-} & m)(t+k)]-[j t+3 m(t+k)] \\
& =m t+(1-m)(t+k)-j t-3 m(t+k) \\
& =t+k-3 m t-4 m k-j t \\
& =t+k-(1-j) t-4 m k-j t \\
& =t+k-t+j t-4 m k-j t \\
& =
\end{aligned}
$$


which is a constant. Thus there will be a constant exposure duration difference between performance curves, as predicted by the additive model.

\section{Proof That Multiplicative Predictions Hold for a Probability-Matching Model}

Assume that attending to the stimulus location increases stimulus encoding rate by some factor, $c$. Assume as before that on some proportion of the trials, $j(j>.5)$, subjects attend to the cued location. If $t$ is the valid-cue exposure duration required to reach some performance level for trials on which subjects attend to the cue, then the average valid-cue exposure duration required to reach any given performance level is

$$
j t+(1-j) t c .
$$

I again assume that when a subject does not attend to the cued location, the subject is equally likely to attend to any of the remaining three locations. Thus for invalid-cue trials, the average exposure duration required for equivalent performance is

$$
\left(\frac{1-j}{3}\right) t+\left[1-\left(\frac{1-j}{3}\right)\right] t c
$$

Again let $m=(1-j) / 3$. Substituting $3 m$ for $1-j$ and $1-3 m$ for $j$, the difference between valid-and invalid-cue exposure durations predicted by the multiplicative model at any performance level is

$$
\begin{aligned}
& {[m t+(1-m) t c]-[(1-3 m) t+3 m t c] } \\
&= m t+(1-m) t c-(1-3 m) t-3 m t c \\
&=4 m t+t c-4 m t c-t \\
&=t(4 m+c-4 m c-1) \\
&=t(c-1)(1-4 m) .
\end{aligned}
$$

Since $c-1$ and $1-4 m$ are both constants, their product, $c^{\prime}$, is also a constant. Thus the predicted horizontal difference between performance curves is

$$
t c^{\prime}
$$

which defines a multiplicative model.

(Manuscript received June 5, 1989;

revision accepted for publication November 30, 1989.) 